\title{
Frequency Sweep Based Sensing Technology for Non-destructive Electrical Resistivity Measurement of Concrete
}

\author{
S. Wickramanayake, K. Thiyagarajan, S. Kodagoda \& L. Piyathilaka \\ iPipes Lab, Centre for Autonomous Systems, University of Technology Sydney, Australia. \\ E-mail: Karthick.Thiyagarajan@uts.edu.au (Corresponding Author)
}

\begin{abstract}
-
Electrical resistivity is an important parameter to be monitored for the conditional assessment and health monitoring of aging and new concrete infrastructure. In this paper, we report the design and development of a frequency sweep based sensing technology for non-destructive electrical resistivity measurement of concrete. Firstly, a sensing system prototype was developed based on the Wenner probe arrangement for the electrical resistivity measurements. This system operates by integrating three major units namely current injection unit, sensing unit and microcontroller unit. Those units govern the overall operations of the sensing system. Secondly, the measurements from the developed unit were compared with the measurements of the commercially available device at set conditions. This experimentation evaluated the measurement performance and demonstrated the effectiveness of the developed sensor prototype. Finally, the influence of rebar and the effect of frequency on the electrical measurements were studied through laboratory experimentation on a concrete sample. Experimental results indicated that the electrical resistivity measurements taken at a closer proximity to the rebar had its influence than the measurements taken away from the rebar in the ideal set condition. Also, the increase in electrical resistivity to the increase in frequency was observed, and then the measurements show lesser variations to higher frequency inputs.
\end{abstract}

\section{Keywords -}

Concrete, electrical resistivity, frequency effects, frequency sweep, infrastructure, infrastructure health monitoring, non-destructive, rebar effects, sensor.

\section{Introduction}

Robotics play a significant role in the condition assessment of water utility assets for evaluating the integrity of pipelines [1]. Although robotic platforms are used for moving inside the pipelines, the key aspect to nondestructive measurements is the development of reliable sensors $[2,3,4,5]$. In this context, this paper presents the design and development of a sensing system that can be used on the moving robotic platforms inside pipelines for infrastructure health monitoring applications.

The work reported in this paper is motivated by the concrete corrosion problem that wastewater utility faces globally [6]. The losses due to corrosion related problems are estimated to be in the order of millions of dollars worldwide [7]. Presently, the water utilities uses a predictive modelling approach to estimate the corrosion throughout the sewer network [8]. However, such models rely on the quality of sensor data $[9,10]$. Surface moisture data is one of the parameter inputs used for improving the prediction of the model $[11,12]$. Our previous investigations had studied the behaviour of concrete moisture conditions and the electrical resistivity measurements, where we employed direct current based electrical resistivity measurements [13] and through invasive approach [14]. Upon in-situ calibration, the electrical resistivity measurements can be used to determine the concrete moisture condition. However, reliable measurement of electrical resistivity is vital for the application motivated.

In this work, we aim to develop a alternating current based sensing system for accurate measurements of concrete electrical resistivity. In this regard, this paper proposes a frequency sweep based technology for nondestructive electrical resistivity measurement of concrete. The major contributions are:

- Development of a physical unit for measuring the concrete electrical resistivity, where the desired frequency can be set in the unit to perform measurements or a frequency sweep can be performed. Then, the measured readings can be stored in the system's data acquisition unit and visualized through the graphical user interface (GUI).

- Performance evaluation of the developed system was conducted by using a commercially available device's measurements as the benchmark readings. This evaluation demonstrates the effectiveness of the proposed system.

- Influence of rebar location and the effects of frequency levels on the electrical resistivity measurements were studied through the laboratory experimentation on a fabricated concrete sample.

For the readers, the rest of this paper is structured as follows: Section 2 describes the development of the sensing system. Section 3 presents the experimental results with analyses and finally, Section 4 concludes the paper by highlighting the key outcomes and future prospects. 


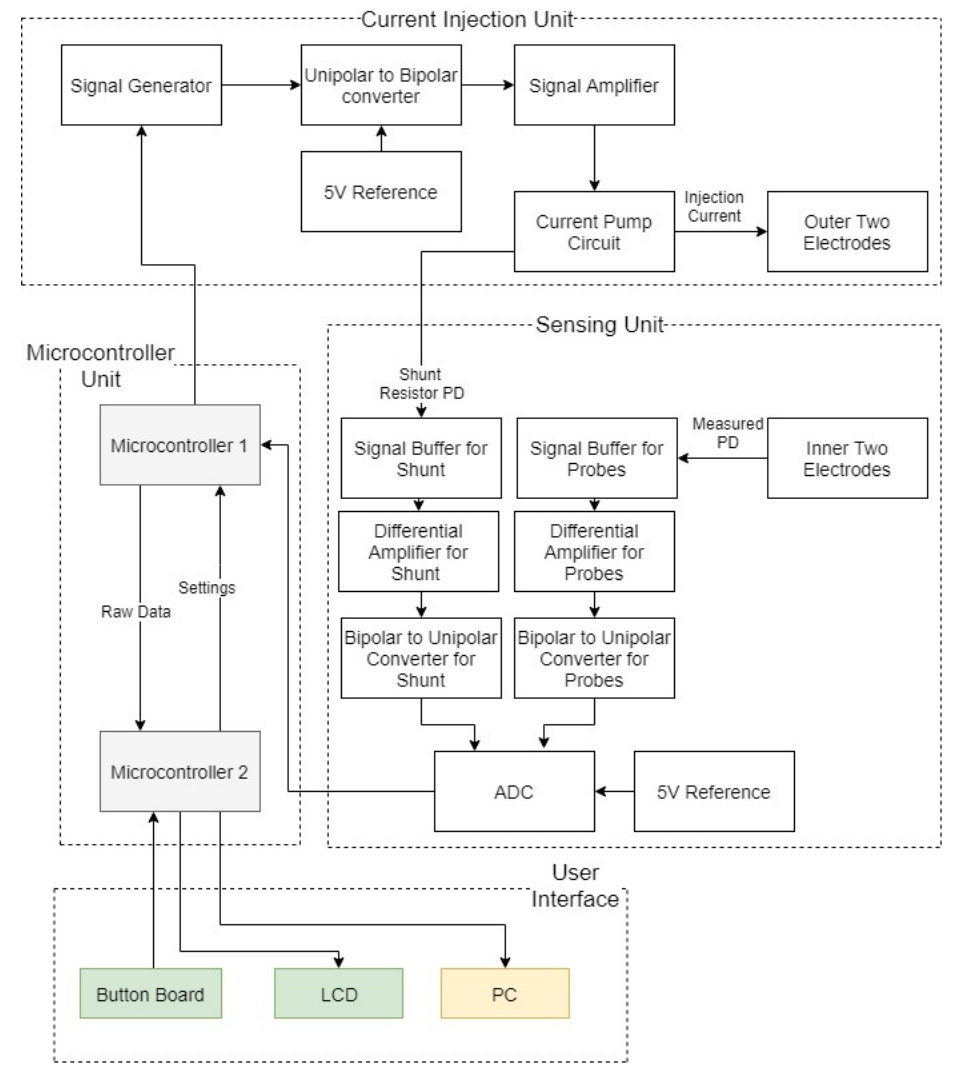

Figure 1. Hardware block diagram.

\section{Development of the Sensing System}

\subsection{Sensing Principle}

The Wenner probe method [15] is used to measure the surface resistivity of the material being tested. It uses four electrodes; outer two electrodes inject an alternating current to the surface while the inner two electrodes measure the potential difference. The principle is true for both DC current and AC Current, but there are some drawbacks when using the DC current. Applying a DC current on to the test material will cause macroscopic polarization, which will create an artificial DC potential on the surface. This will cause the measured potential difference to be inconsistent. This was eliminated by injecting a bipolar AC current in the form of a square wave or a sine wave. The alternating polarity of the injected current will prevent the material from generating an artificial DC potential.

The potential difference measured from the inner two probes and the known injected current can be used to compute the resistance of the material by using the Ohm's Law. The electrical resistivity of the material depends on the resistance and the probe spacing. Therefore, the calculated resistance and the distance between the probes can be used to compute the resistivity of the material based on the Equation (1).

$$
E R=2 \pi a \frac{V}{I}
$$

where $E R$ is the measured electrical resistivity, a $=38 \mathrm{~mm}$ is the spacing between the electrodes, $V$ is the measured potential difference and $I$ is the applied current.

A major component of this design is the ability to vary the frequency of the injected current. The frequency of the injected current affects the penetration depth of the current. Lower frequencies will allow the current to penetrate deeper into the material than the higher frequencies. This characteristic behaviour can be used to determine the resistivity values at certain depths of the material being tested. The developed sensor will allow the user to perform a frequency sweep by setting the maximum and the minimum limits and the stepping frequency. During the frequency sweep, the resistivity is measured simultaneously at the set intervals.

\subsection{Design of Sensing System}

The three main components in designing the sensing system are the current injection unit, sensing unit and the micro-controller unit. The block diagram in Figure 1 shows the information flow through each of the major components of the system. 


\subsubsection{Current Injection Unit}

The current injection unit produces a constant bipolar alternating current with a variable frequency. To generate this current, we used a Howland current pump circuit. The ideal Howland current pump circuit is essentially a voltage controlled current source, which can generate a constant current regardless of the load resistance. This is achieved by changing the voltage across the load accordingly to keep the current through the load constant. However, when the resistance of the load is too high, the voltage required to keep the current constant at the required amount will be higher than the input voltage to the circuit. In such situation, the current across the load will be lower than the expected value. This requires the current to be constantly monitored using a shunt resistor to prevent any errors in the resistivity measurements. The current output of the the Howland current pump circuit can be calculated using the Equation (2), where $I$ is the injected current, $V_{i n}$ is the input voltage and $R_{S}$ is the shunt resistance.

$$
I=\frac{V_{\text {in }}}{R_{S}}=\frac{15}{60000}=250 \mu \mathrm{A}
$$

The output current, which will be injected into the test surface was kept very small in the range of micro-amperes due to several reasons. A lower current eliminates the heat generation on the surface from the injected current, which will cause the resistivity readings to alter. The power loss over transmitting wires is also minimized and any shock hazard from persons coming in contact with the current probes will also be eliminated by using a smaller current.

For producing the alternating current, we have used the analog devices AD9850 Signal Generator IC to generate the square wave. It is an integrated chip that uses advanced Direct Digital Synthesizer (DDS) technology coupled with a high speed 10-bit DAC to produce a sine wave. This sine wave is connected to a square wave using an internal comparator. The frequency of the signal can be digitally programmed for up to $125 \mathrm{MHz}$ with an accuracy of $0.3 \mathrm{~Hz}$. The signal generated from the AD9850 IC is unipolar with the signal alternating between $0 \mathrm{~V}$ and $5 \mathrm{~V}$. The Wenner probe methodology requires a bipolar alternating current to produce the accurate results without generating any artificial potential differences. To achieve this, we have used a uni-polar to bipolar converter, which was designed using a differential op-amp configuration. After identifying the required gain and the offset of the differential amplifier, we came up with the transfer function in Equation (3) for the unipolar to bipolar converter, where $V_{\text {out }}$ is the output voltage and $V_{i n}$ is the input voltage.

$$
V_{\text {out }}=2 \times V_{\text {in }}-5 \mathrm{~V}
$$

The signal output from the uni-polar to bipolar converter has an amplitude of $\pm 5 \mathrm{~V}$ which is not sufficient for measuring resistivity using the Wenner probe method. The signal was amplified using a non-inverting op-amp configuration to an amplitude of $\pm 15 \mathrm{~V}$. The op-amp used in the amplifier circuit had to have the rail-to-rail feature to allow the op-amp to output voltages equal to the supply voltage of $\pm 15 \mathrm{~V}$.

\subsubsection{Sensing Unit}

The sensing unit measures the potential difference between the inner two probes of the Wenner array configuration. The potential difference generated in the inner two probes was first passed through a signal buffer circuit and then converted to a measurable value using a differential amplifier, which is then converted back to a uni-polar signal to be measured by the ADC. There is a high impedance difference between the ADC and the electrodes, which had to be taken into consideration when designing the sensing unit. It is also necessary to isolate the sensing unit with the current injection unit to prevent any errors in the measurements due to the common ground terminals. Those issues were addressed by using the signal buffer circuits. The buffer circuits used were voltage buffer amplifiers, which converts the high output impedance of the sensing electrode to a low impedance output to match the impedance of the ADC. The buffer amplifiers also prevented the sensing unit from loading the sensing electrodes and interfering with the current waves created in the test surface. In addition, they were used while measuring the voltage across the shunt resistors due to the same reasons. The circuit schematic in Figure 2 shows the buffer circuit used between the sensing electrodes and the ADC.

The ADC that we used in the sensing unit was the Microchip MCP3008 10-bit ADC. This ADC contains four pseudo differential channels, which are sufficient as this system requires only two differential inputs for the sensing electrodes and the shunt resistor. The voltage output from the buffer circuits was fed through a differential amplifier before being read by the ADC. Since this ADC accepts only a uni-polar input, the measured potential, which is a bipolar signal has to be converted back to a unipolar signal before being fed in to the ADC. The ADC will be powered using a regulated $5 \mathrm{~V}$ supply and the the reference voltage needed by the ADC will be supplied using a voltage reference circuit consisting of a LM4040 reference voltage IC.

\subsubsection{Microcontroller}

The microcontroller is the central unit of the system, which manages all the other components. An Arduino Micro for the microcontroller was utilized as it supports SPI and $\mathrm{I}^{2} \mathrm{C}$ communication protocol, which is required to communicate with the other components of the system. 


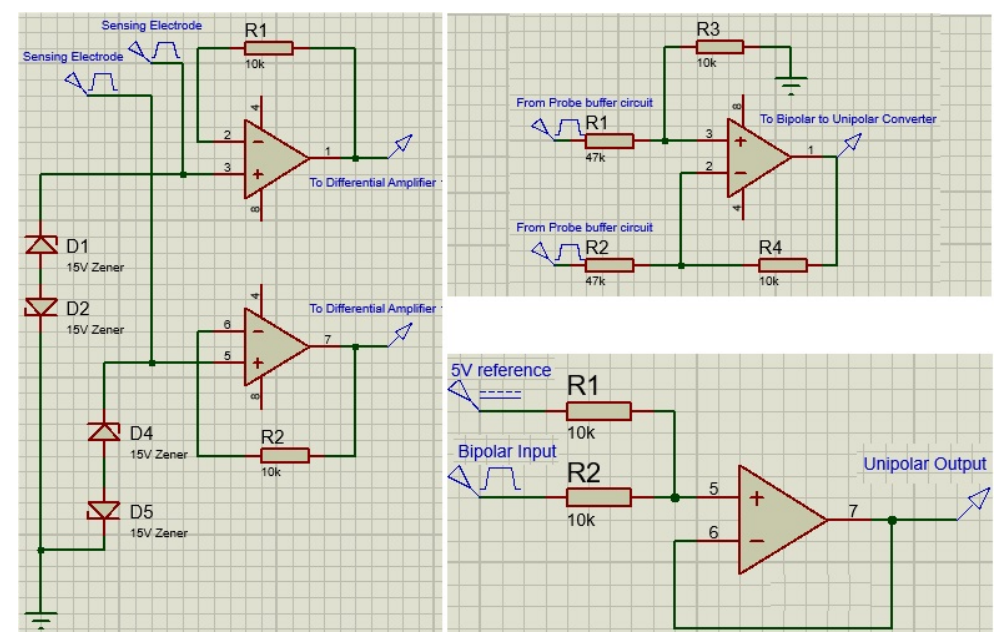

Figure 2. (Left) Signal buffer circuit schematic of the sensing electrodes. (Top Right) Differential amplifier circuit schematic of the sensing electrodes. (Bottom Right) Bipolar to unipolar converter.

The ADC needs to operate constantly without any interruptions during the measurement process. Therefore, it requires the Arduino connected with the ADC not to be interrupted during data logging. To carry out measurements and data logging at the same time, it is necessary to use a second Arduino to handle the data logging and the user interactions. This enables the results to be displayed in real-time while the device is in operation.

\subsection{Operating System}

The microcontroller programming of the system was carried out using the Arduino IDE and the GUI was designed and implemented using the Matlab software. The software was designed to produce useful information by processing the raw data, so that the user can interpret the measured readings easily to determine the health conditions of the structure under investigation. The control unit of this system consists of two microcontroller boards, which contains two different sets of programming instructions. The unit containing the first microcontroller board is referred to as the 'Sensor board' while the unit containing the second microcontroller is referred to as the 'UI board'

The sensor board was programmed to receive the system settings from the user including the information about the required frequency sweep and the data logging. The user information is then used to start the current injection unit, which injects a current though the outer two probes of the sensor. The potential difference measured by the inner two probes is then captured using the ADC in the sensor board. The UI board consists of the buttons, which are used to change the settings of the system and also, a LCD screen to display the settings and the real-time resistivity measurements. The raw data captured from the sensor board is passed on to the UI board for processing and to be presented to the user. The data received from the sensor board is the AC signal, which has been converted to a unipolar signal from the bipolar to unipolar converter. This signal is then converted back to a bipolar signal using software and sent though a set of filters and then, processed to plot a graph of resistivity against the frequency. The digital reading will be shown in the LCD of the UI board and plots for greater data visualization will be shown in a GUI on a computer connected to the sensor via USB. Figure 3 shows the overall software flow.

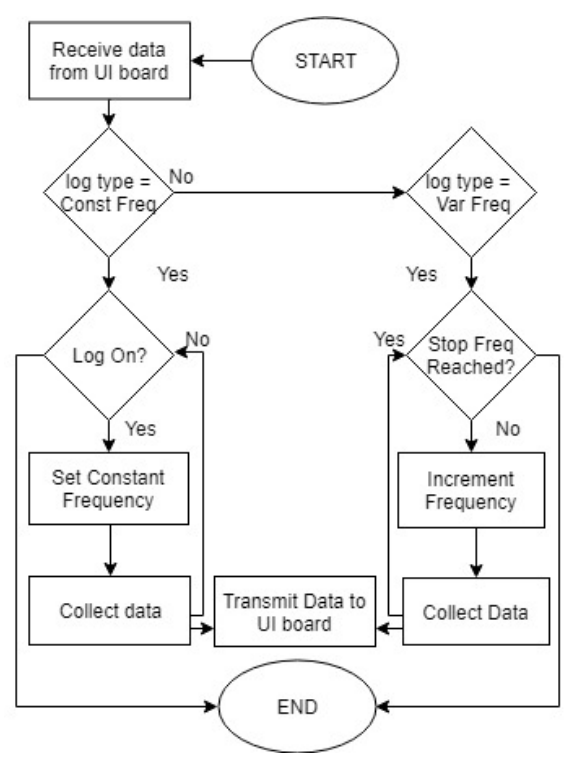

Figure 3. Overall software flow diagram. 


\subsection{Graphical User Interface for Visualization}

The system can be connected to a computer via a USB that allows the results to be visualized graphically through the GUI, which was designed by using the Matlab software (R2016a version), and it interacts with the hardware through serial communication. The GUI allows real-time monitoring of the data and also the system can be completely controlled from the GUI rather than the hardware buttons. The resistivity values will be displayed in the system's GUI along with the frequency of the injected current. The system is programmed to plot the real-time measurements for better visualization of the resistivity variations during the frequency sweep. Further, the GUI offers an option to filter the data to remove any noise signals produced by the sensor. In addition, the GUI can also be used to easily save the logged data into a computer as a .csv or a .txt text file for comprehensive analysis. Figure 4 shows a screenshot of the GUI.

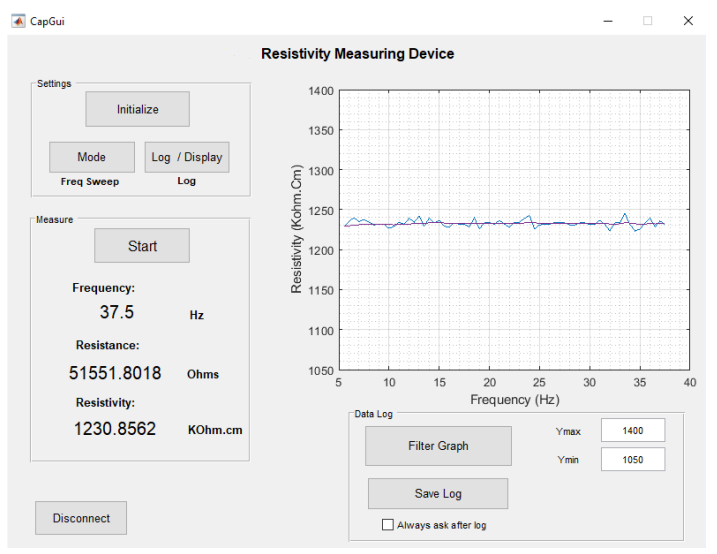

Figure 4. The GUI of the developed system.

\subsection{Electrodes Arrangement and Enclosure Design}

For the electrical resistivity measurement of concrete, the electrodes are required to be in contact with the test surface. A stainless steel 316-grade material bolts were chosen as electrodes mainly because of the reason that they highly resist in corrosive and high humidity environments, and has a good electrical conductivity. The electrodes were housed in a 3D printed enclosure containing a spring mechanism, which allows the electrodes to be pressed on the test surface firmly to allow maximum surface contact. Figure 5 shows the electrode arrangement with housing.

The main circuits including the micro-controller, signal generator, current pump circuit and all the other circuits were housed inside a plastic enclosure as shown in Figure 6, with cut-outs for the buttons, LCD display, power jack, USB port and the mini-DIN type connector that connects the circuits enclosure with the electrodes.

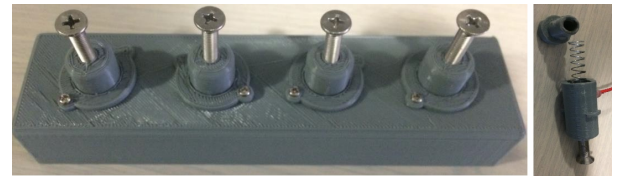

Figure 5. (Left) Assembled electrode arrangement. (Right) Electrode with spring mechanism.

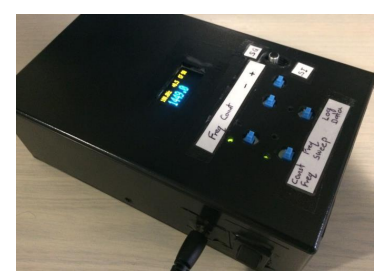

Figure 6. Enclosure of the computing unit.

\section{Experimental Results}

\subsection{Measurement Performance Evaluation}

In this performance evaluation, we have utilized a commercially available electrical resistivity meter (Resipod, PCTE) [16] for the purpose of measurement comparison with the developed unit. The commercial device injects a current of $250 \mu \mathrm{A}$ at a frequency of $40 \mathrm{~Hz}$ during measurements. To represent an identical test condition, we have set our developed system to produce a current of 250 $\mu \mathrm{A}$ at a frequency of $40 \mathrm{~Hz}$. A concrete sample having $10 \mathrm{~cm}$ thickness was fabricated and a rebar having $1 \mathrm{~cm}$ diameter was embedded at $2 \mathrm{~cm}$ distance from the top surface. Using both the devices, five sets of readings were taken on the concrete samples at each location and similar measurements were taken at varying horizontal distances to the rebar as shown in Figure 7.

Table 1 tabulates the electrical resistivity measurements

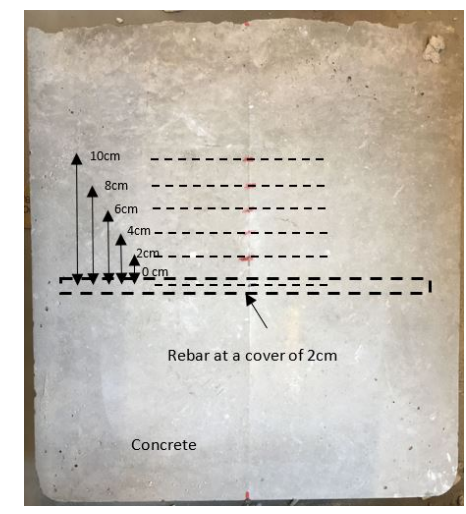

Figure 7. The concrete sample used for testing with embedded rebar and measurements were taken at $2 \mathrm{~cm}$ spacing away from the rebar up to $10 \mathrm{~cm}$. 
from the commercial device and the developed unit. It can be observed that the mean absolute error (MAE) for the measurements taken in different horizontal distances is less than $1 \mathrm{k} \Omega \mathrm{cm}$, which indicates the effective measurement performance of the developed unit.

Table 1. Measurement Performance Evaluation

\begin{tabular}{clll}
\hline $\begin{array}{l}\text { Horizontal } \\
\text { distance to } \\
\text { the rebar } \\
(\mathrm{cm})\end{array}$ & $\begin{array}{l}\text { Mean } \\
\text { electrical } \\
\text { resistivity } \\
\text { from Resipod } \\
(\mathrm{k} \Omega \mathrm{cm})\end{array}$ & $\begin{array}{l}\text { Mean } \\
\text { electrical } \\
\text { Resistivity } \\
\text { from } \\
\text { developed } \\
\text { unit } \\
(\mathrm{k} \Omega \mathrm{cm})\end{array}$ & $\begin{array}{l}\text { Mean } \\
\text { absolute } \\
(\mathrm{k} \Omega \mathrm{cm})\end{array}$ \\
\hline 0 & 3.27 & 3.20 & 0.07 \\
\hline 2 & 8.83 & 9.63 & 0.80 \\
\hline 4 & 10.9 & 10.07 & 0.83 \\
\hline 6 & 13.2 & 12.5 & 0.7 \\
\hline 8 & 16.23 & 16.17 & 0.06 \\
\hline 10 & 17.9 & 17.83 & 0.07 \\
\hline
\end{tabular}

\subsection{Influence of Rebar Location}

In order to study the influence of rebar location on the electrical resistivity measurements, an experimentation was conducted on a concrete sample that has rebar at a known depth. In this experiment, the current of a constant frequency of $80 \mathrm{~Hz}$ was injected to the surface of the concrete sample and the resistivity was calculated using the measured potential difference. A frequency of $80 \mathrm{~Hz}$ was chosen as it is low enough to cause a variation in resistivity due to the rebar, which is located $2 \mathrm{~cm}$ below the concrete surface. The sensor is prone to noise signals during measurements. Therefore, the resistivity readings were taken for a period of time so that an average can be obtained to minimize any error from the noise. The plot in Figure 8 shows the measurements taken on the concrete sample while moving $2 \mathrm{~cm}$ horizontally from the rebar between each measurement.

A mean value for the resistivity was obtained by generating a line of best fit for the measurements during the $200 \mathrm{~ms}$ interval. The constant frequency of $80 \mathrm{~Hz}$ allowed the current to penetrate into the test material upto a certain depth and produce current flux lines. The rebar in the concrete affects the generated current flux lines, which will result in differences in the measured potential difference. The plot in Figure 9 shows the change in resistivity against the sensor distance to rebar, where we can observe that the resistivity increases as the sensor distance to rebar increases. This shows that rebar has an influence on electrical resistivity measurements taken at closer proximity.

\subsection{Effect of Frequency on Measurements}

The frequency of the injected AC current affects the penetration depth of the current flux generated from the

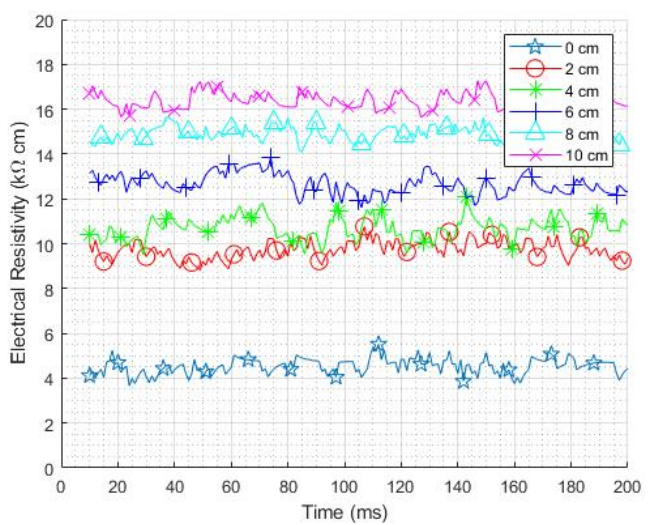

Figure 8 . Resistivity vs Time graph, with the first measurement taken directly above the rebar $(0 \mathrm{~cm})$ and last measurement at $10 \mathrm{~cm}$ away from rebar.

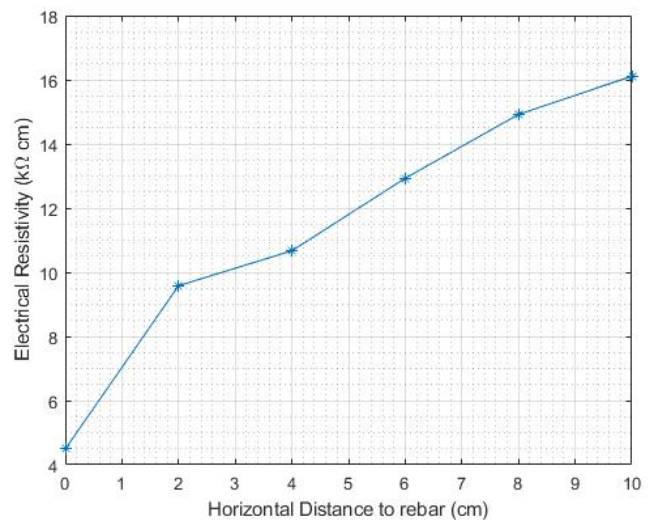

Figure 9. Resistivity vs Horizontal distance to rebar.

outer two probes of the Wenner probe array. An experimentation was conducted, where the frequency sweep was carried out in the frequency range of $1 \mathrm{~Hz}$ to $1.8 \mathrm{KHz}$ with the resolution of $2 \mathrm{~Hz}$. Measurements were taken on a concrete sample of $2 \mathrm{~cm}$ rebar cover and the developed sensing unit was moved $2 \mathrm{~cm}$ away between each set of measurements. The changes in resistivity measurements were plotted against the increase in frequency as shown in Figure 10 in order to analyze the effects due to the frequency sweep of the injected current.

It can be observed from the Figure 10 that there is an increase in resistivity as the frequency increases between $1 \mathrm{~Hz}$ to approximately about $150 \mathrm{~Hz}$ on the measurements taken with the sensor directly above the rebar $(0 \mathrm{~cm})$, and similar trend was was observed with measurements taken at $2 \mathrm{~cm}, 4 \mathrm{~cm}, 6 \mathrm{~cm}, 8 \mathrm{~cm}$ and $10 \mathrm{~cm}$ away from the rebar. This variation is due to the changes in the penetration depth as the frequency of the injected current changes and 


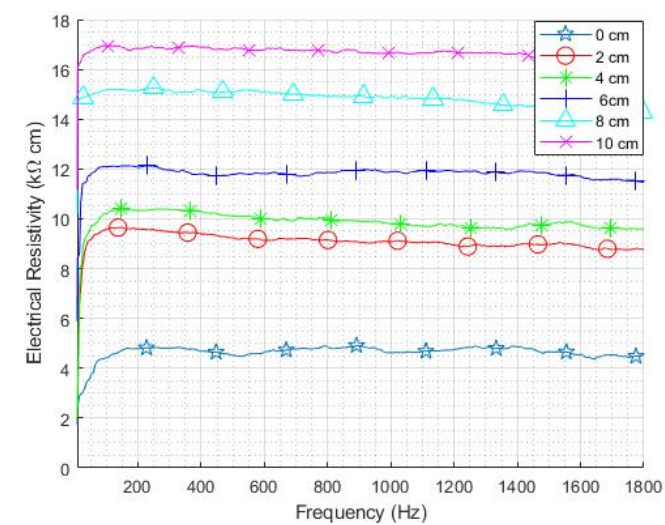

Figure 10. Resistivity variations for frequency changes on a concrete sample with $2 \mathrm{~cm}$ rebar cover, with the first measurement taken directly above the rebar $(0 \mathrm{~cm})$ and moving $2 \mathrm{~cm}$ away from the rebar between each measurement until $10 \mathrm{~cm}$.

the influence due to the presence of rebar in the region of interest of the sensing unit.

The current at lower frequencies has a larger wavelength, which allows for greater penetration. This enables more current flux lines to be created in the concrete, which increases the number of current flux lines interfering with the rebar. This causes the potential difference measured by the inner two probes of the developed unit to be smaller, which translates to a smaller resistivity measurement. Approximately, above $150 \mathrm{KHz}$ frequency, the resistivity readings shows less variations. When the frequency is greater than a certain value, the penetration power of the injected current is too low to penetrate deep enough into the rebar, which eliminates the influence on the resistivity due to the rebar.

The point at which the resistivity measurements starts to exhibit less variations is earlier when the sensor is $2 \mathrm{~cm}$ away from the rebar than when it is directly above the rebar, as shown in Figure 10 and Figure 11. This is due to the variation of the distance between the sensor and the rebar. When the sensor was moved over $4 \mathrm{~cm}$ away from the rebar, a less change of resistivity was observed, because of the reason that the rebar position is now away from the maximum penetration range.

\section{Summary and Future Prospects}

In this paper, we have presented the developments of a frequency sweep based sensing technology for nondestructive concrete electrical resistivity measurements. From the reported work, the key highlights include:

- Development of a sensor prototype for measuring the electrical resistivity of concrete and data acquisition.

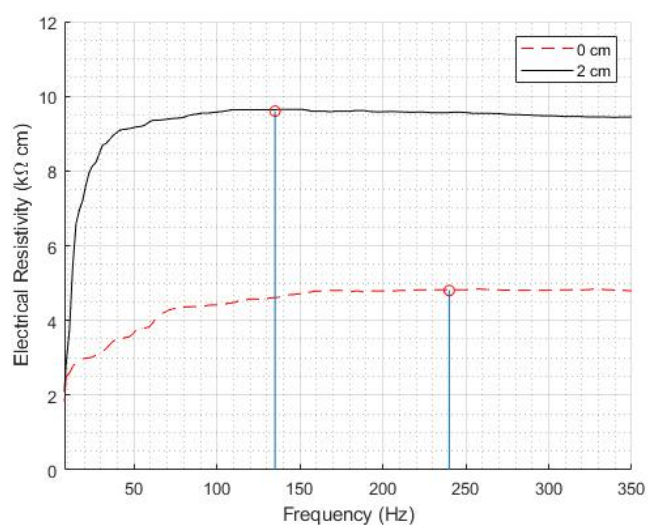

Figure 11. Resistivity versus Frequency graph for the concrete sample with $2 \mathrm{~cm}$ rebar cover, with $0 \mathrm{~cm}$ and $2 \mathrm{~cm}$ plot, close up view that shows the point at which the resistivity converges to lesser variations.

- Measurement results indicated that the MAE between the developed unit and commercial device is less than $1 \mathrm{k} \Omega \mathrm{cm}$, which demonstrates the effectiveness of the prototype.

- Effect of the rebar on the electrical resistivity measurement was studied. Experimentation results showed that the measurements taken at closer proximity to the rebar has lower values than the measurements taken at different distances away from the rebar.

- Effect of the frequency sweep on the electrical resistivity measurement was studied. Measurement results displayed that there is an increasing trend in the electrical resistivity measurements with the increase in frequency approximately upto $150 \mathrm{~Hz}$ and then for higher frequencies, lesser variations in the electrical resistivity was observed.

In the future, the reported sensing system will be improved by integrating with wireless data transfer feature, and can be employed for real-world applications motivated in $[17,18]$.

\section{References}

[1] Jaime Valls Miro, Nalika Ulapane, Lei Shi, Dave Hunt, and Michael Behrens. Robotic pipeline wall thickness evaluation for dense nondestructive testing inspection. Journal of Field Robotics, 2018.

[2] K Thiyagarajan, S Kodagoda, and J K Alvarez. An instrumentation system for smart monitoring of surface temperature. In 2016 14th International Con- 
ference on Control, Automation, Robotics and Vision (ICARCV), pages 1-6, 2016.

[3] Nalika Ulapane, Alen Alempijevic, Jaime Valls Miro, and Teresa Vidal-Calleja. Nondestructive evaluation of ferromagnetic material thickness using Pulsed Eddy Current sensor detector coil voltage decay rate. NDT and E International, 2018.

[4] Linh Nguyen, Nalika Ulapane, Jaime Valls Miro, Gamini Dissanayake, and Fredy Munoz. Improved signal interpretation for cast iron thickness assessment based on pulsed eddy current sensing. In Proceedings of the 2017 12th IEEE Conference on Industrial Electronics and Applications, ICIEA 2017, 2018.

[5] Nalika Ulapane, Linh Nguyen, Jaime Valls Miro, Alen Alempijevic, and Gamini DIssanayake. Designing a pulsed eddy current sensing set-up for cast iron thickness assessment. In Proceedings of the 2017 12th IEEE Conference on Industrial Electronics and Applications, ICIEA 2017, 2018.

[6] Tony Wells, Robert Melchers, Antony Joseph, Phil Bond, Dammika Vitanage, Heriberto Bustamante, John De Grazia, Thomas Kuen, John Nazimek, and Ted Evans. A collaborative investigation of the microbial corrosion of concrete sewer pipe in Australia. In OzWater-12 AustraliaâẮ́s National Water Conference and Exhibition, May, pages 8-10, 2012.

[7] K Thiyagarajan and S Kodagoda. SMART monitoring of surface temperature and moisture content using multisensory data fusion. In 2015 IEEE 7 th International Conference on Cybernetics and Intelligent Systems (CIS) and IEEE Conference on Robotics, Automation and Mechatronics (RAM), pages 222-227, 2015.

[8] Jianjia Zhang, Bin Li, Xuhui Fan, Yang Wang, and Fang Chen. Corrosion prediction on sewer networks with sparse monitoring sites: A case study. In Lecture Notes in Computer Science (including subseries Lecture Notes in Artificial Intelligence and Lecture Notes in Bioinformatics), 2018.

[9] K. Thiyagarajan, S. Kodagoda, and L. Van Nguyen. Predictive analytics for detecting sensor failure using autoregressive integrated moving average model. In Proceedings of the 2017 12th IEEE Conference on Industrial Electronics and Applications, ICIEA 2017, volume 2018-Febru, 2018.
[10] Karthick Thiyagarajan, Sarath Kodagoda, Linh Van Nguyen, and Ravindra Ranasinghe. Sensor Failure Detection and Faulty Data Accommodation Approach for Instrumented Wastewater Infrastructures. IEEE Access, 6:56562-56574, 2018.

[11] Karthick Thiyagarajan, Sarath Kodagoda, Ravindra Ranasinghe, Dammika Vitanage, and Gino Iori. Robust sensing suite for measuring temporal dynamics of surface temperature in sewers. Scientific Reports, 8(1):16020, 122018.

[12] B. Li, X. Fan, J. Zhang, Y. Wang, F. Chen, S. Kodagoda, T. Wells, L. Vorreiter, D. Vitanage, G. Iori, D. Cunningham, and T. Chen. Predictive Analytics Toolkit for H2S Estimation and Sewer Corrosion. In OZWater, Sydney, 2017. Australian Water Association.

[13] K Thiyagarajan, S Kodagoda, and N Ulapane. Datadriven machine learning approach for predicting volumetric moisture content of concrete using resistance sensor measurements. In 2016 IEEE 11th Conference on Industrial Electronics and Applications (ICIEA), pages 1288-1293, 2016.

[14] Karthick Thiyagarajan and Sarath Kodagoda. Analytical Model and Data-driven Approach for Concrete Moisture Prediction. In 33rd International Symposium on Automation and Robotics in Construction (ISARC 2016), pages 298-306, Auburn, 2016. IAARC.

[15] W. Morris, E. I. Moreno, and A. A. Sagüés. Practical evaluation of resistivity of concrete in test cylinders using a Wenner array probe. Cement and Concrete Research, 1996.

[16] Resipod Surface Resistivity Meter. www.forconstructionpros.com, 2011.

[17] Karthick Thiyagarajan. Robust Sensor Technologies Combined with Smart Predictive Analytics for Hostile Sewer Infrastructures. PhD thesis, University of Technology Sydney, 2018.

[18] Karthick Thiyagarajan, Sarath Kodagoda, Linh Van Nguyen, and Sathira Wickramanayake. Gaussian Markov Random Fields for Localizing Reinforcing Bars in Concrete Infrastructure. In 2018 Proceedings of the 35th International Symposium on Automation and Robotics in Construction, pages 1052-1058, Berlin, 2018. International Association for Automation and Robotics in Construction. 